\title{
The four-variable modification of diet in renal disease formula underestimates glomerular filtration rate in obese type 2 diabetic individuals with chronic kidney disease
}

\author{
S. Nair • V. Mishra • K. Hayden • P. J. G. Lisboa • \\ B. Pandya • S. Vinjamuri • K. J. Hardy • \\ J. P. H. Wilding
}

Received: 17 November 2010 / Accepted: 17 January 2011/Published online: 26 February 2011

(C) Springer-Verlag 2011

\begin{abstract}
Aims/hypothesis GFR is commonly estimated using the four-variable Modification of Diet in Renal Disease (MDRD) formula and this forms the basis for classification of chronic kidney disease (CKD). We investigated the effect of obesity on the estimation of glomerular filtration rate in type 2 diabetic participants with $\mathrm{CKD}$.
\end{abstract}

\author{
S. Nair · J. P. H. Wilding $(\bowtie)$ \\ Obesity \& Endocrinology Research Unit, \\ Clinical Sciences Centre, \\ University Hospital Aintree, University of Liverpool, \\ Longmoor Lane, \\ Liverpool L9 7AL, UK \\ e-mail: j.p.h.wilding@liv.ac.uk \\ V. Mishra $\cdot$ K. Hayden \\ Department of Biochemistry, \\ Aintree University Hospitals NHS Foundation Trust, \\ Liverpool, UK
}

P. J. G. Lisboa

Department of Mathematics \& Statistics and Institute for Health

Research, Liverpool John Moores University,

Liverpool, UK

B. Pandya

Department of Nephrology,

Aintree University Hospitals NHS Foundation Trust,

Liverpool, UK

S. Vinjamuri

Department of Nuclear Medicine,

Royal Liverpool University Hospital NHS Trust,

Liverpool, UK

S. Nair $\cdot$ K. J. Hardy

Diabetes \& Endocrinology Department,

St Helens \& Knowsley Teaching NHS Trust,

Liverpool, UK
Methods We enrolled 111 patients with type 2 diabetes mellitus in different stages of CKD. GFR was measured using ${ }^{51} \mathrm{Cr}$-labelled EDTA plasma clearance and was estimated using the four-variable MDRD formula.

Results The bias between estimated and measured GFR was $-22.4(-33.8$ to -11.0$) p<0.001$ in the obese group compared with $-6.04(-17.6$ to -5.5$) p=0.299$ in the nonobese group. When GFR was indexed to body surface area of $1.73 \mathrm{~m}^{2}$, the bias remained significant at $-9.4(-13.4$ to -5.4$)$ $p<0.001$ in the obese participants.

Conclusions/interpretation This study suggests that the four-variable MDRD formula significantly underestimates GFR in obese type 2 diabetic participants with CKD.

Keywords Bias · Chronic kidney disease - Effect of obesity on GFR - Estimated GFR - Glomerular filtration rate .

MDRD equation - Type 2 diabetes mellitus
Abbreviations
BSA Body surface area
CKD Chronic kidney disease
eGFR Estimated glomerular filtration rate
MDRD Modification of diet in renal disease

\section{Introduction}

Nephropathy in diabetes is the leading cause of end-stage renal disease [1] and is associated with significantly increased cardiovascular risk [2-4]. It is important to be able to screen and accurately stage patients with diabetes and chronic kidney disease (CKD) to enable timely intervention to delay progression, as recommended by national and international guidelines $[5,6]$ and to ensure 
that medication to control vascular risks and concomitant conditions is used safely at appropriate doses. GFR is a key indicator of kidney function, but measuring it with inulin clearance or isotopic methods is time consuming, expensive and clinically inapplicable. Hence kidney function is routinely assessed and monitored using creatinine based estimated GFR (eGFR). An equation developed from the Modification of Diet in Renal Disease (MDRD) study [7], the four-variable MDRD formula based on serum creatinine, age, sex and race is currently widely employed [8]. Most biochemistry laboratories now report eGFR on samples sent for measurement of renal function.

The MDRD formula has been shown to underestimate GFR in overweight and obese individuals with newly diagnosed type 2 diabetes mellitus [9]. We speculated that this bias continues into later stages of diabetic CKD. This will have significant implications for the management of patients with type 2 diabetes mellitus, over $85 \%$ of whom are overweight or obese, where treatment choices are influenced by GFR. For example, metformin, a drug with proven cardiovascular benefits to overweight type 2 diabetic patients [10] may be denied owing to GFR underestimation.

The aim of the present study was to estimate the bias between isotopic GFR and eGFR in patients with type 2 diabetes mellitus, and relate it to BMI. In comparison with published studies examining this bias, our study population comprised participants with CKD stages 1 to 4 , which include the particularly important category of individuals with eGFR between 30 and 60 , for whom treatment decisions are often determined by an eGFR cut-off value. Also, unlike in the early CKD stages, a bias in the more advanced CKD stages will have significant clinical implications.

Indexing GFR to a body surface area (BSA) of $1.73 \mathrm{~m}^{2}$ in obese patients has been shown to significantly reduce the GFR, and some authors have suggested that unindexed GFR is preferable in routine practice and clinical studies for the obese population [11-13]. We report both GFR and GFR indexed to BSA of $1.73 \mathrm{~m}^{2}$ (indexed GFR) and also examine the difference between them in the obese and nonobese categories.

\section{Methods}

Study population The study population comprised 111 white participants with type 2 diabetes mellitus associated CKD, with baseline characteristics as presented in Table 1 . We employed a purposive quota sampling method from a hospital diabetes clinic population to achieve the required numbers in each group. The study was approved by the local research ethics committee and all participants gave written informed consent.

Serum creatinine assay and eGFR calculation Serum creatinine was measured using the kinetic alkaline picrate assay on an Olympus AU640 analyser (Olympus, Watford, UK). The modified MDRD equation, traceable to isotope dilution mass spectrometry (IDMS)-creatinine and adjusted

Table 1 Demographics and bias between eGFR and measured GFR

\begin{tabular}{lccc}
\hline Variable & All participants & BMI $\leq 30 \mathrm{~kg} / \mathrm{m}^{2}$ & $\mathrm{BMI}>30 \mathrm{~kg} / \mathrm{m}^{2}$ \\
\hline$n$ & 111 & 37 & 74 \\
Male/female & $56 / 55$ & $32 / 14$ & $33 / 41$ \\
Age (years) & $65.7 \pm 10.7(66.0[58.0-74.0])$ & $69.3 \pm 11.2(72[63-77])$ & $63.9 \pm 10.0(64.0[57.0-73.0])$ \\
Weight $(\mathrm{kg})$ & $90.74 \pm 22.4(87.9[73.9-102.8])$ & $71.9 \pm 11.5(71.0[63.1-80.3])$ & $100 \pm 20.6(97.2[86.1-112.7])$ \\
BMI $\left(\mathrm{kg} / \mathrm{m}^{2}\right)$ & $33.0 \pm 7.3(32.3[27.7-37.5])$ & $25.3 \pm 2.8(25.9[23.2-27.9])$ & $36.8 \pm 5.6(35.1[32.3-40.3])$ \\
BSA $\left(\mathrm{m}^{2}\right)$ & $1.97 \pm 0.25(1.95[1.7-2.1])$ & $1.81 \pm 0.17(1.82[1.6-1.9])$ & $2.05 \pm 0.24(2.02[1.89-2.21])$ \\
HbA $1 \mathrm{c}(\%)$ & $8.3 \pm 1.6(8.1[7.1-9.4])$ & $8.7 \pm 1.7(8.3[7.4-9.5])$ & $8.1 \pm 1.5(8.0[6.8-9.3])$ \\
Creatinine $(\mu \mathrm{mol} / \mathrm{l})$ & $107.6 \pm 31.8(98.0[87-126])$ & $103 \pm 23.3(99.0[87.5-113.0])$ & $109.6 \pm 35.2(97.5[84.5-128.0])$ \\
GFR $\left(\mathrm{ml} / \mathrm{min}^{2}\right)$ & $84.2 \pm 38.9(74.5[55.5-106.9])$ & $74.6 \pm 24.7(71.4[54.9-86.9])$ & $89.0 \pm 43.7(77.0[57.4-110.6])$ \\
Indexed GFR & $73.1 \pm 28.4(72.3[51.1-87.7])$ & $67.3 \pm 21.1(64.3[51.4-83.3])$ & $76.0 \pm 31.2(74.6[48.2-91.5])$ \\
$\left(\mathrm{ml} \mathrm{min}{ }^{-1} 1.73 \mathrm{~m}^{-2}\right)$ & $67.2 \pm 24.3(65.8[48.2-85.5])$ & $68.6 \pm 23.0(69.4[52.9-87.2])$ & $66.6 \pm 25.0(62.8[47.0-85.8])$ \\
eGFR $\left(\mathrm{ml} \mathrm{min}{ }^{-1} 1.73 \mathrm{~m}^{-2}\right)$ & $-16.9(-8.4,-25.0) p<0.001$ & $-6.04(-17.6,-5.5) p=0.299$ & $-22.4(-33.8,-11) p<0.001$ \\
Bias $(95 \% \mathrm{CI})$ & $-5.82(-9.3,-2.3) p=0.01$ & $1.38(-7.7,5.0) p=0.66$ & $-9.4(-13.1,-5.4) p<0.001$ \\
Bias $1(95 \% \mathrm{CI})$ & & &
\end{tabular}

Data are means $\pm \mathrm{SD}$ (median [interquartile range]), except for bias figures

GFR is the isotopically measured GFR

Bias is the mean difference between eGFR and GFR. Bias1 is the mean difference between eGFR and indexed GFR 
for Olympus methodology $\left(\mathrm{ml} \mathrm{min} \min ^{-1} 1.73 \mathrm{~m}^{-2}\right)$, is as follows:

$$
\begin{aligned}
175 & \times\{[(\text { serum creatinine }-16.14) / 0.955] \times 0.011312\}^{-1.154} \\
& \times(\text { age })^{-0.203} \times(0.742 \text { if female }) \times(1.212 \text { if Afro-Caribbean })
\end{aligned}
$$

was used to calculate eGFR [14].

GFR measurement We used the ${ }^{51} \mathrm{Cr}$-labelled EDTA clearance method, which has been validated against the gold standard inulin clearance method $[15,16]$. A bolus of 3.0 to $3.5 \mathrm{MBq}{ }^{51} \mathrm{Cr}$-labelled EDTA was administered to the participants intravenously at $0 \mathrm{~min}$ and the slopeintercept GFR was measured from three samples taken at 120, 180 and $240 \mathrm{~min}$ using a Cobra II Auto-Gamma Sample counter (PerkinElmer, Waltham, MA, USA). This was corrected for one-pool approximation using the quadratic Brøchner-Mortensen equation [17]. GFR was indexed to BSA $\left[\mathrm{GFR}_{\text {corrected }}=\mathrm{GFR}_{\text {measured }} \times(1.73 /\right.$ BSA $\left.\mathrm{m}^{2}\right)$ ] and expressed as millilitres per minute $1.73 \mathrm{~m}^{-2}$. BSA was calculated based on the DuBois and DuBois formula: BSA $\left(\mathrm{m}^{2}\right)=\left[\right.$ weight $\left.(\mathrm{kg})^{0.425}\right] \times[$ height $\left.(\mathrm{cm})^{0.725}\right] \times 0.007184[18]$.

Statistical analysis Statistical analysis was carried out using SPSS (version 17.0 for Windows; SPSS, Chicago, IL, USA). The four-variable MDRD eGFR results were compared with GFR by means of two-tailed paired $t$ tests or the Wilcoxon signed-rank test as appropriate. We defined bias as the mean of the difference between eGFR and GFR, so it was negative when eGFR underestimated GFR. Results are presented as mean $\pm \mathrm{SD}$ unless otherwise indicated and $p<$ 0.05 was taken to indicate statistical significance.

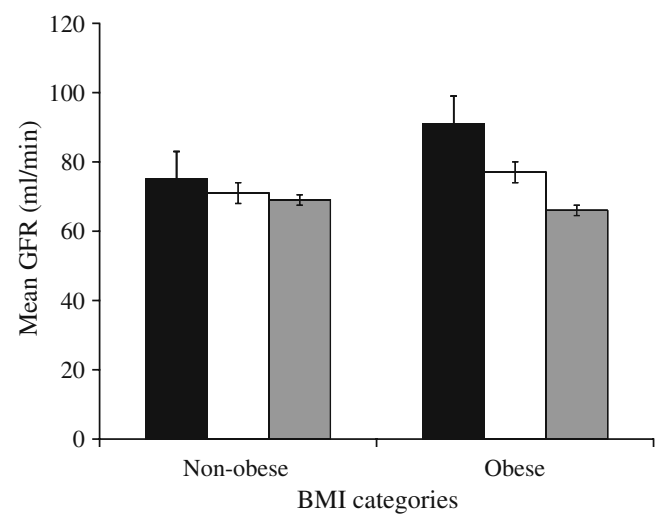

Fig. 1 Bias between GFR, indexed GFR and eGFR in type 2 diabetic participants with CKD in the obese and non-obese categories. The figure also shows the significant reduction in GFR when it is indexed to BSA in the obese population. Black bar, GFR; white bar, indexed GFR; grey bar, eGFR. The error bars represent standard error

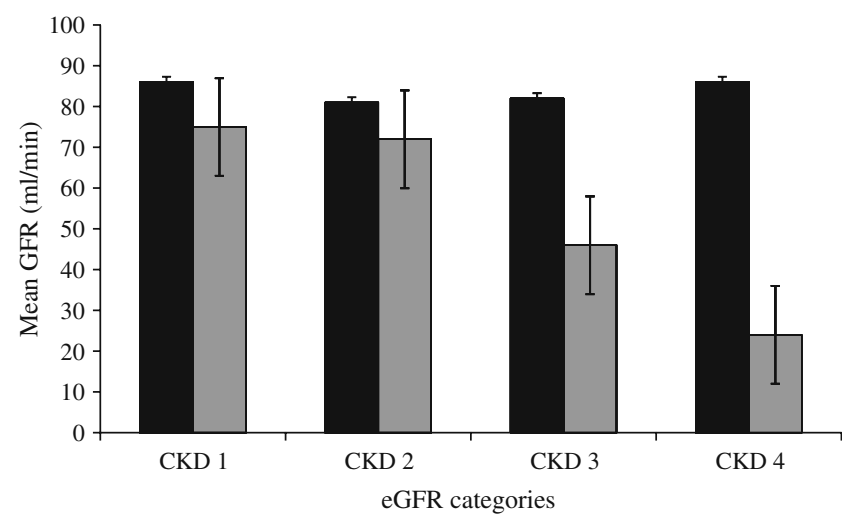

Fig. 2 Trend of increasing bias between eGFR and GFR in CKD stages $1-4$ in obese participants with type 2 diabetes. The bias attained statistical significance in CKD stage 3. Black bar, GFR; grey bar, eGFR. The error bars represent standard error

\section{Results}

Table 1 provides the descriptive statistics of the study population. The four-variable MDRD formula significantly underestimates GFR in obese patients with type 2 diabetes mellitus as shown in Fig. 1. A significant bias persisted into CKD stage 3 in the obese (BMI $>30)$ participants as shown in Fig. 2.

The bias between eGFR and GFR translates to a misclassification of study participants in the obese category into higher CKD groups. Forty per cent of participants in CKD stage 2 were misclassified into stage 3 and $54 \%$ from stage $3 \mathrm{~A}$ to stage $3 \mathrm{~B}$.

Analysis of bias by sex shows a mean overall difference between women and men of $-16.6(95 \% \mathrm{CI}-30.6,-2.6, p=$ $0.02)$; in the obese participants the difference was -13.7 (95\% CI $-32.6,5.02, p=0.148)$ and in non-obese participants the difference was $-18.60(95 \% \mathrm{CI}-39.3,2.17, p=0.078)$.

It was also noted that the mean BSA increased with increasing BMI and was $2.05 \pm 0.24 \mathrm{~m}^{2}$ in the obese participants. The mean difference between the GFR and the indexed GFR was $13.02 \pm 53.2(p=0.039)$ in the obese participants. This discrepancy between eGFR and GFR before and after indexing to a BSA of $1.73 \mathrm{~m}^{2}$ can also be visualised from Fig. 1.

\section{Discussion}

This study extends previous observations made in recently diagnosed patients with normal renal function [9], by demonstrating that the four-variable MDRD formula underestimates GFR in obese patients with established type 2 diabetes mellitus. Furthermore, we have found that this bias between eGFR and GFR persists into later stages of CKD. The bias was clinically significant in CKD stage 4 , but not 
statistically so owing to the low number of participants in this group. Overall, the bias appeared to be greater in women, which could be due to the fact that women tend to have significantly higher body fat content and lower muscle mass compared with men. The difference in bias across sex was similar in magnitude, but did not reach significance in either the obese or non-obese groups analysed separately; however, the study was not powered to look at sex differences.

Underestimated GFR will have implications for the management of obese patients with type 2 diabetes mellitus, particularly as the choice of drugs, such as metformin and some of the newer glucose lowering drugs, is influenced by eGFR. More than half of our obese participants with eGFR $>$ 45 were misclassified into a more severe CKD stage by the four-variable MDRD equation. This could lead to earlier than necessary initiation of insulin in these patients with possible adverse effects, including weight gain and hypoglycaemia. As we move away from a glucocentric approach to managing basic pathophysiology in type 2 diabetes [19], obese patients may be denied important therapeutic options because of an underestimated GFR.

This study also highlights the problem with indexing measured GFR as it is currently recommended in the obese population. Indexing is based on the assumption that the average BSA is $1.73 \mathrm{~m}^{2}$, but it is much higher in obese individuals. While this practice of indexing does not cause major differences in non-obese individuals, it leads to significant underestimation of measured GFR in obese individuals.

There is a need for better equations for estimating GFR in obese individuals, which are validated against the gold standard isotopic GFR in this population. Until such an equation becomes available, clinicians should consider this bias when making treatment decisions in obese patients with type 2 diabetes.

Duality of interest The authors declare that there is no duality of interest associated with this study. No external funding was obtained for this study.

\section{References}

1. Molitch ME, DeFronzo RA, Franz MJ et al (2004) Nephropathy in diabetes. Diab Care 27(Suppl 1):S79-S83

2. Valmadrid CT, Klein R, Moss SE, Klein BEK (2000) The risk of cardiovascular disease mortality associated with microalbuminuria and gross proteinuria in persons with older-onset diabetes mellitus. Arch Intern Med 160:1093-1100

3. Mattock MB, Morrish NJ, Viberti G, Keen H, Fitzgerald AP, Jackson G (1992) Prospective study of microalbuminuria as predictor of mortality in NIDDM. Diabetes 41:736-741

4. Buckley BS, Simpson CR, McLernon DJ, Murphy AW, Hannaford PC (2009) Five year prognosis in patients with angina identified in primary care: incident cohort study. BMJ 339:b3058

5. KDOQI (2007) KDOQI clinical practice guidelines and clinical practice recommendations for diabetes and chronic kidney disease. Am J Kidney Dis 49:S12-S154

6. RCP (2006) Joint Specialty Committee on Renal Medicine of the Royal College of Physicians of London and the Renal Association: Chronic kidney disease in adults; UK guidelines for identification, management and referral. Available from www. renal.org/CKDguide/full/CKDprintedfullguide.pdf

7. Levey AS, Bosch JP, Lewis JB, Greene T, Rogers N, Roth D (1999) A more accurate method to estimate glomerular filtration rate from serum creatinine: a new prediction equation. Modification of Diet in Renal Disease Study Group. Ann Intern Med 130:461-470

8. Stevens LA, Coresh J, Greene T, Levey AS (2006) Assessing kidney function-measured and estimated glomerular filtration rate. N Engl J Med 354:2473-2483

9. Chudleigh RA, Dunseath G, Peter R et al (2008) Influence of body weight on the performance of glomerular filtration rate estimators in subjects with type 2 diabetes. Diab Care 31:47-49

10. UKPDS (1998) Effect of intensive blood-glucose control with metformin on complications in overweight patients with type 2 diabetes (UKPDS 34). Lancet 352:854-865

11. Delanaye P, Radermecker RP, Rorive M, Depas G, Krzesinski JM (2005) Indexing glomerular filtration rate for body surface area in obese patients is misleading: concept and example. Nephrol Dial Transplant 20:2024-2028

12. Delanaye P, Mariat C, Cavalier E, Krzesinski JM (2009) Indexing glomerular filtration rate for body surface area: myth and reality. Néphrol Thér 5:614-622

13. Peters AM, Glass DM (2010) Use of body surface area for assessing extracellular fluid volume and glomerular filtration rate in obesity. Am J Nephrol 31:209-213

14. Reynolds TM, Twomey PJ (2007) Implications of method specific creatinine adjustments on General Medical Services chronic kidney disease classification. J Clin Pathol 60:1048-1050

15. Ditzel J, Vestergaard P, Brinklov M (1972) Glomerular filtration rate determined by ${ }^{51} \mathrm{Cr}$-EDTA-Complex. A practical method based upon the plasma disappearance curve determined from four plasma samples. Scand J Urol Nephrol 6:166-170

16. Brøchner-Mortensen J (1985) Current status on assessment and measurement of glomerular filtration rate. Clin Physiol Funct Imaging 5:1-17

17. Fleming JS, Zivanovic MA, Blake GM, Burniston M, Cosgriff PS; British Nuclear Medicine Society (2004) Guidelines for the measurement of glomerular filtration rate using plasma sampling. Nucl Med Commun 25:759-769

18. Dubois D, Dubois EF (1915) The measurement of the surface area of man. Arch Intern Med 15:868-881

19. Defronzo RA (2009) Banting Lecture. From the triumvirate to the ominous octet: a new paradigm for the treatment of type 2 diabetes mellitus. Diabetes 58:773-795 